PROCEEDINGS OF THE

AMERICAN MATHEMATICAL SOCIETY

Volume 139, Number 10, October 2011, Pages 3513-3525

S 0002-9939(2011)10789-5

Article electronically published on February 17, 2011

\title{
MULTIDIMENSIONAL CHROMATIC DERIVATIVES AND SERIES EXPANSIONS
}

\author{
ALEKSANDAR IGNJATOVIC AND AHMED I. ZAYED
}

(Communicated by Walter Van Assche)

\begin{abstract}
Chromatic derivatives and series expansions of bandlimited functions have recently been introduced as an alternative representation to the Taylor series, and they have been shown to be more useful in practical signal processing applications than in the Taylor series. Although chromatic series were originally introduced for bandlimited functions, they have now been extended to a larger class of functions. The $n$-th chromatic derivative of an analytic function is a linear combination of the $k$-th ordinary derivatives with $0 \leq k \leq n$, where the coefficients of the linear combination are based on a suitable system of orthogonal polynomials. The goal of this article is to extend chromatic derivatives and series to higher dimensions. This is of interest not only because the associated multivariate orthogonal polynomials have much reacher structure than in the univariate case, but also because we believe that the multidimensional case will find natural applications to fields such as image processing and analysis.
\end{abstract}

\section{INTRODUCTION}

The Paley-Wiener space, $P W_{\pi}$, of functions bandlimited to $[-\pi, \pi]$ consists of square integrable functions whose Fourier transforms have supports in $[-\pi, \pi]$. It is also known that functions in $P W_{\pi}$, are entire functions of exponential type at most $\pi$ that are square integrable when restricted to the real axis. A standard series representation of any function $f \in P W_{\pi}$ is given by the Whittaker-ShannonKotel'nikov (WSK) [17] sampling series

$$
f(t)=\sum_{n=-\infty}^{\infty} f(n) \frac{\sin \pi(t-n)}{\pi(t-n)} .
$$

This expansion may be viewed as a global expansion because it uses function values at infinitely many points uniformly distributed on the real line. On the other hand, as an entire function, $f$ has a Taylor series expansion of the form $f(t)=$ $\sum_{n=0}^{\infty}\left(f^{(n)}(0) / n !\right) t^{n}$, which may be viewed as a local expansion since it uses the values of $f$ and all its derivatives at a single point. Unlike the sampling series, which plays an important role in signal processing, the Taylor series has very limited applications because, among other reasons, a truncated Taylor series is a polynomial and is not bandlimited.

Received by the editors January 24, 2010 and, in revised form, August 24, 2010.

2010 Mathematics Subject Classification. Primary 41A58, 42C15; Secondary 94A12, 94A20.

Key words and phrases. Chromatic derivatives, chromatic expansions, signal representation.

(C)2010 American Mathematical Society

Reverts to public domain 28 years from publication 
Chromatic derivatives and series expansions have recently been introduced by A. Ignjatovic in [8, 9] as an alternative representation to the Taylor series, and they have been shown to be more useful in practical applications than the Taylor series; see [5, 6, 17, 12, 13, 14, 15].

The $n$-th chromatic derivative $K^{n}[f]\left(t_{0}\right)$ of an analytic function $f(t)$ at $t_{0}$ is a linear combination of the ordinary derivatives $f^{(k)}\left(t_{0}\right), 0 \leq k \leq n$, where the coefficients of the combination are based on systems of orthogonal polynomials.

Chromatic derivatives are intrinsically related to the Fourier transformation. They are constructed using the fact that, under the Fourier transformation, differentiation in the time domain corresponds to multiplication by powers of $\omega$ in the frequency domain.

For the reader's convenience, we will briefly describe how chromatic series are constructed. Let $W(\omega)$ be a non-negative weight function such that all of its moments are finite, i.e., such that

$$
\mu_{n}=\int_{-\infty}^{\infty} \omega^{n} W(\omega) d \omega<\infty .
$$

Let $\left\{P_{n}(\omega)\right\}$ be the family of polynomials orthonormal with respect to $W(\omega)$,

$$
\int_{-\infty}^{\infty} P_{n}(\omega) P_{m}(\omega) W(\omega) d \omega=\delta(m-n),
$$

and let $K^{n}(f)=P_{n}\left(-i \frac{d}{d t}\right)(f)$ be the corresponding linear differential operators obtained from $P_{n}(\omega)$ by replacing $\omega^{k}(0 \leq k \leq n)$ with $\left(-i \frac{d}{d t}\right)^{k} 11$ These differential operators are called chromatic derivatives associated with the family of orthogonal polynomials $\left\{P_{n}(\omega)\right\}$ because they preserve the spectral features of a bandlimited signal. They can be evaluated with high accuracy and in a noise robust way from samples of the signal taken at a small multiple of the usual Nyquist rate; see [11] and [10] for details.

Let $\psi(z)$ be the Fourier transform of the weight function $W(\omega)$,

$$
\psi(z)=\int_{-\infty}^{\infty} e^{i \omega z} W(\omega) d \omega .
$$

Such $\psi(z)$ will be used in a Taylor-type expansion of functions analytic in a domain around the origin. Since $\psi^{(n)}(0)=i^{n} \mu_{n}, \psi(z)$ is analytic around the origin whenever $\lim \sup \left(\mu_{n} / n !\right)^{1 / n}<\infty$. As shown in [1], this condition holds if and only if

$$
\int_{-\infty}^{\infty} e^{c|\omega|} W(\omega) d \omega<\infty
$$

for some $c>0$, and in this case $\psi(z)$ is analytic in a strip $S_{d}(c / 2)=\left\{z: \Im(z)<\frac{c}{2}\right\}$.

The chromatic expansion of a function $f \in C^{\infty}$ is the formal series

$$
f(z) \sim \sum_{n=0}^{\infty} K^{n}(f)(u) K^{n}(\psi)(z-u) .
$$

It has been shown in 11] that if $f(z)$ is analytic in the $\operatorname{strip} S_{d}(c / 2)$ and if $\sum_{n=0}^{\infty}\left|K^{n}(f)(0)\right|^{2}$ converges, then for all $u \in \mathbb{R}$, the series (1.1) converges to $f(z)$ uniformly in every $\operatorname{strip}\{z:|\Im(z)|<c / 2-\varepsilon\}$, for every $\varepsilon>0$. Here it

\footnotetext{
${ }^{1}$ In some of our previous work the chromatic derivatives were defined as $K^{n}=(-i)^{n} P^{n}\left(i \frac{d}{d t}\right)$, which ensures that chromatic derivatives of real functions are real. The definition used in this paper results in simpler formulas.
} 
should be emphasized that although chromatic series were originally introduced for bandlimited functions, the theory now applies to a much larger class of functions.

In the particular case, where $W(\omega)=\chi_{(-1,1)}$, the chromatic series associated with the Legendre polynomials converge in the whole complex plane, i.e., the strip $S_{d}(c / 2)$ is $\mathbb{C}$, and the set of entire functions for which $\sum_{n=0}^{\infty}\left|K^{n}(f)(0)\right|^{2}$ converges is precisely the set of $L^{2}$ functions whose Fourier transforms are finitely supported, i.e., the set of bandlimited functions. For such functions the chromatic expansions converge uniformly on $\mathbb{R}$, and their truncations are themselves bandlimited. This is in contrast to the Taylor series, whose truncations are not bandlimited. For that reason the chromatic series have practical significance for signal processing. The chromatic series can be defined for more general cases of operator polynomials; see [18.

The goal of this article is to extend chromatic derivatives and series to higher dimensions. Although applications of multidimensional chromatic series have not been established yet, we hope that this article will lay the theoretical foundations for such applications. One of the key points in the treatment of multidimensional chromatic series is to find the optimum orthogonal polynomial system that will best fit the shape of the support of the Fourier transform of a bandlimited multidimensional signal.

The article is organized as follows. In Section 2, we give a brief account of the theory of orthogonal polynomials in several variables with emphasis on the results that we will need in the following section. The main results are presented in Section 3, with examples in the following sections.

\section{Orthogonal POLYNOMIALS IN SEVERAL VARIABLES}

Let $N_{0}$ denote the set of nonnegative integers and $\alpha$ be a multi-index $\alpha=$ $\left(\alpha_{1}, \ldots, \alpha_{d}\right) \in N_{0}^{d}$. We use the notation

$$
\alpha !=\alpha_{1} ! \alpha_{2} ! \ldots \alpha_{d} !, \quad|\alpha|=\alpha_{1}+\ldots+\alpha_{d},
$$

and $\delta_{\alpha, \beta}=\delta_{\alpha_{1}, \beta_{1}} \ldots \delta_{\alpha_{d}, \beta_{d}}$. For $x=\left(x_{1}, \ldots, x_{d}\right) \in \mathbb{R}^{d}$, we define the monomial $x^{\alpha}=x_{1}^{\alpha_{1}} \ldots x_{d}^{\alpha_{d}}$, and $|\alpha|$ is the degree of $x^{\alpha}$. A polynomial $P$ in $d$ variables is a linear combination of the form

$$
P(x)=\sum_{\alpha} c_{\alpha} x^{\alpha}
$$

where $c_{\alpha}$ are complex numbers. We denote the set of all polynomials in $d$ variables by $\Pi^{d}$ and the set of all polynomials of degree at most $n$ by $\Pi_{n}^{d}$. The set of all homogenous polynomials of degree $n$ will be denoted by $P_{n}^{d}$ :

$$
P_{n}^{d}=\left\{P: P(x)=\sum_{|\alpha|=n} c_{\alpha} x^{\alpha}\right\} .
$$

Every polynomial in $d$ variables can be written as a linear combination of homogenous polynomials

$$
P(x)=\sum_{k=0}^{n} \sum_{|\alpha|=k} c_{\alpha} x^{\alpha} .
$$


It is known that the dimension $r_{n}^{d}$ of $P_{n}^{d}$ is

$$
r_{n}^{d}=\left(\begin{array}{l}
n+d-1 \\
n
\end{array}\right) .
$$

For a fixed $d$ we may write $r_{n}=r_{n}^{d}$. In one variable, monomials are ordered according to their degrees as $1, x, x^{2}, \ldots$; however, in several variables such natural order does not exist. Therefore, we will use the lexicographic order, i.e., $\alpha>\beta$ if the first non-zero entry in the difference $\alpha-\beta=\left(\alpha_{1}-\beta_{1}, \alpha_{2}-\beta_{2}, \ldots, \alpha_{d}-\beta_{d}\right)$ is positive.

If $\langle$,$\rangle is an inner product on \Pi^{d}$, we say that a polynomial $P$ is orthogonal to a polynomial $Q$ if $\langle P, Q\rangle=0$. A polynomial $P$ is called an orthogonal polynomial if $P$ is orthogonal to all polynomials of lower degree; i.e., $\langle P, Q\rangle=0$ for all $Q \in \Pi^{d}$ with $\operatorname{deg} Q<\operatorname{deg} P$. Denote by $V_{n}^{d}$ the space of orthogonal polynomials of degree exactly $n$ :

$$
V_{n}^{d}=\left\{P \in \Pi_{n}^{d}:\langle P, Q\rangle=0, \text { for all } Q \in \Pi_{n-1}^{d}\right\} .
$$

The dimension of $V_{n}^{d}$ is the same as that of $P_{n}^{d}$.

A multisequence $s: N_{0}^{d} \rightarrow \mathbb{R}$ is written as $s=\left(s_{\alpha}\right)_{\alpha \in N_{0}^{d}}$, and for each multisequence we define a linear functional on $\Pi^{d}$ by

$$
\mathcal{L}\left(x^{\alpha}\right)=s_{\alpha}, \quad \alpha \in N_{0}^{d}
$$

Let the elements of the set $\left\{\alpha \in N_{0}^{d}:|\alpha|=n\right\}$ be arranged as $\alpha^{(1)}, \alpha^{(2)}, \ldots, \alpha^{\left(r_{n}\right)}$ according to the lexicographic order. Let $\mathbf{x}^{\mathbf{n}}$ denote the column vector

$$
\mathbf{x}^{\mathbf{n}}=\left(\mathbf{x}^{\alpha}\right)_{|\alpha|=\mathbf{n}}=\left(\mathbf{x}^{\alpha(\mathbf{j})}\right)_{\mathbf{j}=\mathbf{1}}^{\mathbf{r}_{\mathbf{n}}}
$$

That is, $\mathbf{x}^{\mathbf{n}}$ is a vector whose elements are the monomials $x^{\alpha}$ for $|\alpha|=n$, arranged in the lexicographic order.

Define the vector moments $\mathbf{s}_{\mathbf{k}}=\mathcal{L}\left(\mathbf{x}^{\mathbf{k}}\right)$ and

$$
\mathbf{s}_{(\mathbf{k})+(\mathbf{j})}=\mathcal{L}\left(\mathrm{x}^{\mathbf{k}}\left(\mathrm{x}^{\mathbf{j}}\right)^{\mathbf{T}}\right)
$$

which is a matrix of size $r_{k}^{d} \times r_{j}^{d}$ and has elements $\mathcal{L}\left(x^{\alpha+\beta}\right)$ for $|\alpha|=k,|\beta|=j$, because $\mathbf{x}^{\mathbf{k}}\left(\mathbf{x}^{\mathbf{j}}\right)^{\mathbf{T}}$ is a matrix of size $r_{k}^{d} \times r_{j}^{d}$ whose elements are monomials of $x^{\alpha+\beta}$ with $|\alpha|=k,|\beta|=j$.

Define the matrix

$$
M_{n, d}=\left(s_{(k)+(j)}\right)_{k, j=0}^{n} \text { and } \Delta_{n, d}=\operatorname{det} M_{n, d} ;
$$

$M_{n, d}$ is called a moment matrix and its elements are $\mathcal{L}\left(x^{\alpha+\beta}\right)$ for $|\alpha| \leq n,|\beta| \leq n$. If $\left\{P_{\alpha}\right\}_{|\alpha|=n}$ is a sequence of polynomials in $\Pi_{n}^{d}$, we get the column polynomial vector $\mathbb{P}_{n}=\left(P_{\alpha(1)}, \ldots, P_{\alpha\left(r_{n}\right)}\right)^{T}$, where $\alpha^{(1)}, \ldots, \alpha^{\left(r_{n}\right)}$ is the lexicographic order in $\left\{\alpha \in N_{0}^{d}:|\alpha|=n\right\}$. For more details on the subsequent discussion, see [1, 2$]$.

Definition 2.1. Let $\mathcal{L}$ be a moment functional. A sequence of polynomials $\left\{P_{\alpha}\right\}_{|\alpha|=n}$ in $\Pi_{n}^{d}$ is said to be orthogonal with respect to $\mathcal{L}$ if

$$
\mathcal{L}\left(\mathbf{x}^{\mathbf{m}} \mathbb{P}_{\mathbf{n}}^{\mathbf{T}}\right)=0 \text { for } n>m \text {, and } \mathcal{L}\left(\mathbf{x}^{\mathbf{n}} \mathbb{P}_{\mathbf{n}}^{\mathbf{T}}\right)=s_{n},
$$

where $s_{n}$ is an invertible matrix of size $r_{n}^{d} \times r_{n}^{d}$. 
By definition $\mathcal{L}\left(\mathbf{x}^{\mathbf{m}} \mathbb{P}_{\mathbf{n}}^{\mathbf{T}}\right)=0$ if and only if $\mathcal{L}\left(x^{\beta} P_{\alpha}\right)=0,|\beta|=m,|\alpha|=n$. Hence, each $P_{\alpha}$ is orthogonal to any polynomial of lower degree. It is known that if $\mathcal{L}$ is a moment functional and $\mathbb{P}_{n}$ is orthogonal as defined before, then $\left\{\mathbb{P}_{0}, \mathbb{P}_{1}, \ldots, \mathbb{P}_{n}\right\}$ is a basis for $\Pi_{n}^{d}$. Hence, there exist matrices $\mathbf{c}_{k}$ of size $r_{n}^{d} \times r_{k}^{d}$ such that

$$
\mathbf{x}^{n}=\mathbf{c}_{n} \mathbb{P}_{n}+\mathbf{c}_{n-1} \mathbb{P}_{n-1}+\ldots+\mathbf{c}_{0} \mathbb{P}_{0} .
$$

It is also known that for a given moment functional $\mathcal{L}$, a system of orthogonal polynomials exists if and only if $\Delta_{n, d} \neq 0$.

A moment linear functional $\mathcal{L}$ is said to be positive definite if $\mathcal{L}\left(p^{2}\right)>0$ for all $p \in \Pi^{d}, p \neq 0$. The associated sequence $\left\{s_{\alpha}\right\}$ with $\mathcal{L}$ will also be called positive definite.

If $p=\sum a_{\alpha} x^{\alpha}$, then $\mathcal{L}(p)=\sum a_{\alpha} s_{\alpha}$ and $\mathcal{L}\left(p^{2}\right)=\sum_{\alpha, \beta} a_{\alpha} a_{\beta} s_{\alpha+\beta}>0$ for every sequence $a=\left(a_{\alpha}\right)$ for which $a_{\alpha}=0$, except for finitely many multi-indices $\alpha$. This implies that $\mathcal{L}$ is positive definite if and only if for every tuple $\left(\beta^{(1)}, \ldots, \beta^{(r)}\right)$, $1 \leq j \leq r$, the matrix $\left(s_{\beta^{(i)}+\beta^{(j)}}\right)_{i, j=1}^{r}$ has positive determinant.

It is known that if $\mathcal{L}$ is positive definite, then $\Delta_{n, d}>0$ and there exists a system of orthogonal polynomials with respect to $\mathcal{L}$. In fact, in this case there exists an orthonormal basis with respect to $\mathcal{L}$; that is, there exists a sequence of vector polynomials $\left\{\mathbb{P}_{n}\right\}$ such that

$$
\mathcal{L}\left(\mathbb{P}_{m} \mathbb{P}_{n}^{T}\right)=0 \text { if } m \neq n, \quad \mathcal{L}\left(\mathbb{P}_{n} \mathbb{P}_{n}^{T}\right)=I_{r_{n}}
$$

where $I_{r_{n}}$ is the identity matrix of size $r_{n}^{d} \times r_{n}^{d}$.

Let $M$ denote the set of non-negative Borel measures on $\mathbb{R}^{d}$ having moments of all orders. Thus, $\mu \in M$ if

$$
\int_{\mathbb{R}^{d}}|x|^{\alpha} d \mu<\infty \text { for all } \alpha \in N_{0}^{d}
$$

and we call

$$
s_{\alpha}=\int_{\mathbb{R}^{d}} x^{\alpha} d \mu
$$

the moments of $\mu$. For such a measure $\mu \in M$, we have a moment functional $\mathcal{L}$ defined for polynomials $P \in \Pi^{d}$ by

$$
\mathcal{L}(P)=\int_{\mathbb{R}^{d}} P(x) d \mu(x) .
$$

If $d \mu(x)=W(x) d x$ and $W(x)$ is a non-negative weight function, then $\mathcal{L}$ is positive definite; i.e., $\mathcal{L}\left(P^{2}\right)>0$ for any $0 \neq P \in \Pi^{d}$. It is known that if $s=\left(s_{\alpha}\right)$ is a multisequence, then there exists $\mu \in M$ such that $s_{\alpha}=\int x^{\alpha} d \mu(x)$ if and only if the associated moment functional $\mathcal{L}$ is non-negative on the set of non-negative polynomials. That is, $\mathcal{L}(P) \geq 0$ for any $P \in \Pi_{+}^{d}=\left\{P \in \Pi^{d}: P(x) \geq 0\right\}$.

Let $\mathcal{L}$ be a positive moment function (hence positive definite) and let $\left\{\mathbb{P}_{n}\right\}$ be the system of orthonormal polynomials associated with it. From the above it follows that there exists a measure $\mu \in M$ such that

$$
\mathcal{L}\left(x^{\alpha}\right)=s_{\alpha}=\int_{\mathbb{R}^{d}} x^{\alpha} d \mu(x)
$$

We assume that $\mu$ is absolutely continuous so that $d \mu=W(x) d x$, with $W$ being non-negative so that $\mathcal{L}_{W}(f)=\int f(x) W(x) d x$ is positive definite. We also assume 
that for some $c>0$,

$$
\int_{\mathbb{R}^{d}} e^{c\|x\|} W(x) d x<\infty,
$$

so that polynomials are dense in $L^{2}(d \mu)$, 1, p. 74]. Condition (2.2) is satisfied, for example, if $\mu$ is compactly supported.

We adopt the following notation. If $P\left(x_{1}, \ldots, x_{d}\right)$ is a polynomial in $x_{1}, \ldots, x_{d}$, then the polynomial $P\left(\frac{\partial}{\partial x_{1}}, \ldots, \frac{\partial}{\partial x_{d}}\right)$ will be denoted by $P\left(\frac{\partial}{\partial x}\right)$, where $x^{\alpha}$ is replaced by $\frac{\partial^{|\alpha|}}{\partial x^{\alpha}}$ where $\alpha$ is a multi-index. More explicitly, if $\alpha=\left(\alpha_{1}, \ldots, \alpha_{d}\right)$, then

$$
x^{\alpha}=x_{1}^{\alpha_{1}} x_{2}^{\alpha_{1}} \ldots x_{d}^{\alpha_{d}}, \quad|\alpha|=\alpha_{1}+\alpha_{2}+\ldots+\alpha_{d}
$$

is replaced by

$$
\frac{\partial^{|\alpha|}}{\partial x_{1}^{\alpha_{1}} \partial x_{2}^{\alpha_{2}} \ldots \partial x_{d}^{\alpha_{d}}} .
$$

Let $\left\{P_{\alpha}^{n}\right\}_{n=0}^{\infty}$ denote the sequence of orthonormal polynomials with respect to $\mathcal{L}_{W}$. Let $L_{W}^{2}\left(\mathbb{R}^{d}\right)$ denote the space of all square integrable functions with respect to the weight function $W$.

For any function $f \in L_{W}^{2}\left(\mathbb{R}^{d}\right)$, consider its generalized Fourier expansion with respect to $\left\{P_{\alpha}^{n}\right\}_{n=0}^{\infty}$ :

$$
f \backsim \sum_{n=0}^{\infty} \sum_{|\alpha|=n} c_{\alpha}^{n}(f) P_{\alpha}^{n}, \quad \text { with } \quad c_{\alpha}^{n}(f)=\left\langle f, P_{\alpha}^{n}\right\rangle_{W}=\int f(x) P_{\alpha}^{n}(x) W(x) d x .
$$

If we use the vector notation we have

$$
f \backsim \sum_{n=0}^{\infty} \mathbf{c}_{n}^{T}(f) \mathbb{P}_{n}, \quad \text { with } \quad \mathbf{c}_{n}(f)=\left\langle f, \mathbb{P}_{n}\right\rangle_{W}=\int f(x) \mathbb{P}_{n}(x) W(x) d x ;
$$

here $\mathbf{c}_{n}$ is a column vector with components $c_{\alpha}^{n}$ and $|\alpha|=n$. If we denote the space of orthogonal polynomials of degree exactly $n$ by $V_{n}^{d}$, it follows that (2.3) can be written as

which leads to

$$
f \backsim \sum_{n=0}^{\infty} \operatorname{Proj}_{V_{n}^{d}} f, \quad \text { where } \quad \operatorname{Proj}_{V_{n}^{d}} f=\mathbf{c}_{n}^{T}(f) \mathbb{P}_{n},
$$

$$
\operatorname{Proj}_{V_{n}^{d}} f(x)=\int f(y) \mathbf{P}_{n}(x, y) W(x) d x, \quad \text { with } \quad \mathbf{P}_{n}(x, y)=\mathbb{P}_{n}^{T}(x) \mathbb{P}_{n}(y) .
$$

\section{Multidimensional Chromatic Derivatives}

Let $z=\left(z_{1}, z_{2}, \ldots, z_{d}\right) \in \mathbb{C}^{d}$ and define the inner product

$$
\langle z, w\rangle=\sum_{k=1}^{d} z_{k} \bar{w}_{k}, z, w \in \mathbb{C}^{d} \text { so that }\|z\|^{2}=\sum_{k=1}^{d}\left|z_{k}\right|^{2} .
$$

If $z_{k}=x_{k}+i y_{k}$, define $\Re z=\left(x_{1}, x_{2}, \ldots, x_{d}\right)$ and $\Im z=\left(y_{1}, y_{2}, \ldots, y_{d}\right)$ so that $z=\Re z+i \Im z$. Moreover,

$$
\|\Re z\|^{2}=\sum_{k=1}^{d} x_{k}^{2}, \quad\|\Im z\|^{2}=\sum_{k=1}^{d} y_{k}^{2} .
$$

For every real $a>0$ we let $S_{d}(a)=\left\{z \in \mathbb{C}^{d}:\|\Im(z)\|<a\right\}$. 
Definition 3.1. Let $f: \mathbb{C}^{d} \rightarrow \mathbb{C} t$. The $n$-th chromatic derivatives $\mathbb{K}_{\alpha}^{n}(f)$ of $f(z)$ with respect to the polynomials $\left\{P_{\alpha}^{n}\right\}$ is defined as

$$
\mathbb{K}^{n}(f)=\mathbb{P}_{n}\left(-i \frac{\partial}{\partial z}\right)(f),
$$

where $\mathbb{P}_{n}$ is the column vector defined before.

Hence, the $n^{\text {th }}$ chromatic derivative of $f$ is a column vector with $r_{n}^{d}$ components, with each component a linear combination of partial derivatives. It is easy to check that if $\omega \in \mathbb{R}^{d}$ is fixed, then

$$
K_{\alpha}^{n}\left(e^{i\langle\omega, z\rangle}\right)=P_{\alpha}^{n}(\omega) e^{i\langle\omega, z\rangle} .
$$

Proposition 3.2. Let $\varphi(\omega) \in L_{W}^{2}\left(\mathbb{R}^{d}\right)$ and define a corresponding function $f_{\varphi}$ : $S_{d}(c / 2) \rightarrow \mathbb{C}$ by a $W(\omega)$-weighted inverse Fourier transform of $\varphi$ :

$$
f_{\varphi}(z)=\int_{\mathbb{R}^{d}} \varphi(\omega) e^{i\langle\omega, z\rangle} W(\omega) d \omega .
$$

Then $f_{\varphi}(z)$ is analytic on $S_{d}(c / 2)$, and for all $n$ and $z \in S_{d}(c / 2)$,

$$
\mathbb{K}^{n}\left[f_{\varphi}\right](z)=\int_{\mathbb{R}^{d}} \mathbb{P}_{n}(\omega) \varphi(\omega) e^{i\langle\omega, z\rangle} W(\omega) d \omega .
$$

Proof. Let $z \in \mathbb{C} t^{d}$ and $z=x+i y$, where $x, y \in \mathbb{R}^{d}$, with $\|y\|<c / 2-\varepsilon$ for some $\varepsilon>0$. Then for all $\alpha \in N_{0}^{d}$

$$
\begin{aligned}
& \int_{\mathbb{R}^{d}}\left|(i \omega)^{\alpha} \varphi(\omega) e^{i\langle\omega, z\rangle}\right| W(\omega) d \omega \\
& \leq\left(\int_{\mathbb{R}^{d}}|\varphi(\omega)|^{2} W(\omega) d \omega \int_{\mathbb{R}^{d}} \omega^{2 \alpha} e^{2(\omega \cdot y)} W(\omega) d \omega\right)^{1 / 2} \\
& \quad \leq\|\varphi\|_{W}\left(\int_{\mathbb{R}^{d}} \omega^{2 \alpha} e^{2\|\omega\|\|y\|} W(\omega) d \omega\right)^{1 / 2} .
\end{aligned}
$$

Choose $M_{\alpha}>0$ such that for all $\omega$,

$$
\omega^{2 \alpha}=\omega_{1}^{2 \alpha_{1}} \ldots \omega_{k}^{2 \alpha_{k}} \leq\|\omega\|^{2 \alpha_{1}} \ldots\|\omega\|^{2 \alpha_{k}}=\|\omega\|^{2|\alpha|}<M_{\alpha} e^{\varepsilon\|\omega\|} .
$$

Then

$$
\int_{\mathbb{R}^{d}}\left|(i \omega)^{\alpha} \varphi(\omega) e^{i\langle\omega, z\rangle}\right| W(\omega) d \omega \leq M_{\alpha}^{1 / 2}\|\varphi\|_{W}\left(\int_{\mathbb{R}^{d}} e^{\|\omega\|(c-\varepsilon)} W(\omega) d \omega\right)^{1 / 2}<\infty .
$$

The claims now follow from (2.1), (2.2) and (3.1).

Lemma 3.3. If condition (2.2) holds, then $\left\{\mathbb{P}_{n}(\omega)\right\}$ is a complete system in $L_{W}^{2}\left(\mathbb{R}^{d}\right)$.

Proof. Assume that some $\varphi \in L_{W}^{2}\left(\mathbb{R}^{d}\right)$ is orthogonal to all polynomials $P_{\alpha}^{n}$; i.e., for all $\alpha$,

$$
\int_{\mathbb{R}^{d}} \varphi(\omega) P_{\alpha}^{n}(\omega) W(\omega) d \omega=0
$$

Then (2.1) implies that for all $\alpha$ also

$$
\int_{\mathbb{R}^{d}} \varphi(\omega) \omega^{\alpha} W(\omega) d \omega=0
$$

\footnotetext{
${ }^{2}$ If $x, \omega \in \mathbb{R}^{d}$ are both real vectors, then we denote their scalar product by $x . \omega$. If at least one of $u, z$ is complex, we denote their scalar product by $\langle u, z\rangle$.
} 
Let $f_{\varphi}(z): S_{d}(c / 2) \rightarrow \mathbb{C} t$ be given by (3.2). By Proposition 3.2, $f_{\varphi}$ is analytic on $S_{d}(c / 2)$ and (3.4) implies that $\frac{\partial^{|\alpha|}}{\partial z^{\alpha}} f_{\varphi}(0)=0$ for all $\alpha$. Thus, $f_{\varphi}(z)$ is identically zero on $S_{d}(c / 2)$. This implies that $\varphi(\omega)=0$ almost everywhere on the support of $W(\omega)$; i.e., $\left\{\mathbb{P}_{n}(\omega)\right\}$ is a complete system in $L_{W}^{2}(\mathbb{R})$.

Proposition 3.4. Let $\varphi(\omega) \in L_{W}^{2}\left(\mathbb{R}^{d}\right)$. If for some fixed $u \in S_{d}(c / 2)$ the function $\varphi(\omega) e^{i\langle\omega, u\rangle}$ belongs to $L_{W}^{2}\left(\mathbb{R}^{d}\right)$, then in $L_{W}^{2}\left(\mathbb{R}^{d}\right)$ we have

$$
\varphi(\omega) e^{i\langle\omega, u\rangle}=\sum_{n=0}^{\infty}\left[\mathbb{K}^{n}\left[f_{\varphi}\right](u)\right]^{T} \mathbb{P}_{n}(\omega),
$$

and for $f_{\varphi}$ given by (3.2) we have

$$
\sum_{n=0}^{\infty} \sum_{|\alpha|=n}\left|K_{\alpha}^{n}\left[f_{\varphi}\right](u)\right|^{2}=\left\|\varphi(\omega) e^{i\langle\omega, u\rangle}\right\|_{W}^{2}<\infty .
$$

Proof. Let $f_{\varphi}$ be given by (3.2); by Proposition 3.2. equation (3.3) also holds. However, if $\varphi(\omega) e^{i\langle\omega, u\rangle}$ belongs to the space $L_{W}^{2}\left(\mathbb{R}^{d}\right)$, then $(3.3)$ asserts that the projection of $\varphi(\omega) e^{i\langle\omega, u\rangle}$ onto the vector $\mathbb{P}_{n}(\omega)$ is equal to $\mathbb{K}^{n}\left[f_{\varphi}\right](u)$ :

$$
\left\langle\varphi(\omega) e^{i\langle\omega, u\rangle}, \mathbb{P}_{n}(\omega)\right\rangle_{W}=\mathbb{K}^{n}\left[f_{\varphi}\right](u) .
$$

Since $\left\{\mathbb{P}_{n}(\omega)\right\}_{n \in \mathbb{N}}$ is a complete orthonormal system in $L_{W}^{2}\left(\mathbb{R}^{d}\right)$, (3.7) implies (3.5), and Parseval's Theorem implies (3.6).

Corollary 3.5. For every $\varphi(\omega) \in L_{W}^{2}\left(\mathbb{R}^{d}\right)$ and every $u \in \mathbb{R}^{d}$, equality (3.5) holds and

$$
\sum_{n=0}^{\infty} \sum_{|\alpha|=n}\left|K_{\alpha}^{n}\left[f_{\varphi}\right](u)\right|^{2}=\|\varphi(\omega)\|_{W}^{2} .
$$

Thus, the sum $\sum_{n=0}^{\infty} \sum_{|\alpha|=n}\left|K_{\alpha}^{n}\left[f_{\varphi}\right](u)\right|^{2}$ is independent of $u \in \mathbb{R}^{d}$.

Proof. If $u \in \mathbb{R}^{d}$, then $\varphi(\omega) e^{i\langle\omega, u\rangle} \in L_{W}^{2}\left(\mathbb{R}^{d}\right)$ and $\left\|\varphi(\omega) e^{i\langle\omega, u\rangle}\right\|_{W}^{2}=\|\varphi(\omega)\|_{W}^{2}$; thus, Proposition 3.4 applies.

For one-dimensional examples of the above corollary, see [11].

Definition 3.6. We set for $z \in S_{d}(c / 2)$

$$
\psi(z)=\int_{\mathbb{R}^{d}} e^{i\langle\omega, z\rangle} W(\omega) d \omega,
$$

and more generally,

$$
K_{\alpha}^{n}(\psi(z))=\psi_{\alpha}^{n}(z)=\int_{\mathbb{R}^{d}} P_{\alpha}^{n}(\omega) e^{i\langle\omega, z\rangle} W(\omega) d \omega .
$$

We may also use the vector notation $\boldsymbol{\Psi}_{n}(z)=\left(\psi_{\alpha}^{n}(z)\right)_{|\alpha|=n}$.

Note that Proposition 3.2 implies that the integrals in the definitions of $\psi(z)$ and $\psi_{\alpha}^{n}(z)$ are finite.

Corollary 3.7. Let $\varepsilon>0$. Then for all $z \in S\left(\frac{c}{2}-\varepsilon\right)$

$$
\sum_{n=0}^{\infty} \sum_{|\alpha|=n}\left|\psi_{\alpha}^{n}(z)\right|^{2}<\left\|e^{\left(\frac{c}{2}-\varepsilon\right)\|\omega\|}\right\|_{W}^{2}<\infty .
$$


Proof. We apply Proposition 3.4 with $\varphi(\omega)=1$, in which case $f_{\varphi}(z)=\psi(z)$, and, using (3.6), we obtain

$$
\sum_{n=0}^{\infty} \sum_{|\alpha|=n}\left|\psi_{\alpha}^{n}(z)\right|^{2}=\left\|e^{i\langle\omega, z\rangle}\right\|_{W}^{2}=\left\|e^{|\omega . \Im(z)|}\right\|_{W}^{2} \leq\left\|e^{\|\omega\|\|\Im(z)\|}\right\|_{W}^{2}<\left\|e^{\left(\frac{c}{2}-\varepsilon\right)\|\omega\|}\right\|_{W}^{2} .
$$

The claim now follows from (2.2).

Definition 3.8. We denote by $\Lambda_{W}^{2}$ the vector space of functions $f: S_{d}(c / 2) \rightarrow \mathbb{C}$ which are analytic on $S_{d}(c / 2)$ and satisfy $\sum_{n=0}^{\infty} \sum_{|\alpha|=n}\left|K_{\alpha}^{n}\left[f_{\varphi}\right](0)\right|^{2}<\infty$.

Proposition 3.9. The mapping

$$
f(z) \mapsto \varphi_{f}(\omega)=\sum_{n=0}^{\infty}\left[\mathbb{K}^{n}[f](0)\right]^{T} \mathbb{P}_{n}(\omega)
$$

is an isomorphism between the vector spaces $\Lambda_{W}^{2}$ and $L_{W}^{2}\left(\mathbb{R}^{d}\right)$, and its inverse is given by (3.2).

Proof. Let $f \in \Lambda_{W}^{2}$. Since $\sum_{n=0}^{\infty} \sum_{|\alpha|=n}\left|K_{\alpha}^{n}\left[f_{\varphi}\right](0)\right|^{2}<\infty$, the function $\varphi_{f}(\omega)$ defined by (3.12) belongs to $L_{W}^{2}\left(\mathbb{R}^{d}\right)$. By Proposition [3.2, $f_{\varphi_{f}}$ defined from $\varphi_{f}$ by (3.2) is analytic on $S_{d}(c / 2)$ and it satisfies $\varphi_{f}(\omega)=\sum_{n=0}^{\infty} \mathbb{K}^{n}\left[f_{\varphi_{f}}\right](0) \mathbb{P}_{n}(\omega)$ by Proposition 3.4. By the uniqueness of the Fourier expansion of $\varphi_{f}(\omega)$ with respect to the system $\left\{\mathbb{P}_{n}(\omega)\right\}$, we have $\mathbb{K}^{n}[f](0)=\mathbb{K}^{n}\left[f_{\varphi_{f}}\right](0)$ for all $n$. This, together with (2.1), implies that $\frac{\partial^{|\alpha|}}{\partial x^{\alpha}} f(0)=\frac{\partial^{|\alpha|}}{\partial x^{\alpha}} f_{\varphi_{f}}(0)$; thus, $f(z)=f_{\varphi_{f}}(z)$ for all $z \in S_{d}(c / 2)$. The other direction follows from Propositions 3.2 and 3.4 .

Definition 3.10. For $f \in \Lambda_{W}^{2}$, we denote the corresponding $\varphi_{f}(\omega)$ given by (3.12) by $\mathcal{F}_{W}[f](\omega)$. Thus, for $z \in S_{d}(c / 2)$,

$$
f(z)=\int_{\mathbb{R}^{d}} \mathcal{F}_{W}[f](\omega) e^{i\langle\omega, z\rangle} W(\omega) d \omega .
$$

Proposition 3.9 and Corollary 3.5 imply the following corollary.

Corollary 3.11. For all $f \in \Lambda_{W}^{2}$ and all $x \in \mathbb{R}^{d}$, the sum $\sum_{n=0}^{\infty} \sum_{|\alpha|=n}\left|K_{\alpha}^{n}\left[f_{\varphi}\right](x)\right|^{2}$ converges and is independent of $x$.

3.1. Uniform convergence of chromatic expansions. The chromatic series expansion of a function $f \in C^{\infty}$ is given by the following formal series:

$$
f(z) \sim \sum_{n=0}^{\infty}\left[\mathbb{K}^{n}(f)(u)\right]^{T} \Psi_{n}(z-u) .
$$

The Shannon expansion of a band limited signal is obtained by representing its Fourier transform $\widehat{f}(\omega)$ as a series of trigonometric polynomials. Similarly, the chromatic expansion of an $f \in \Lambda_{W}^{2}$ is obtained by representing $\mathcal{F}_{W}[f](\omega)$ as a series of orthogonal polynomials $P_{\alpha}^{n}(\omega)$.

Proposition 3.12. Assume $f \in \Lambda_{W}^{2}$. Then for all $u \in \mathbb{R}^{d}$ and $\varepsilon>0$, the chromatic series of $f(z)$ converges to $f(z)$ uniformly on $S\left(\frac{c}{2}-\varepsilon\right)$.

Proof. Using (3.10), we get that for all $z \in S_{d}(c / 2)$,

$$
\sum_{k=0}^{n}\left[\mathbb{K}^{k}[f](u)\right]^{T} \Psi_{k}(z-u)=\int_{\mathbb{R}^{d}} \sum_{k=0}^{n}\left[\mathbb{K}^{k}[f](u)\right]^{T} \mathbb{P}_{k}(\omega) e^{i\langle\omega, z-u\rangle} W(\omega) d \omega .
$$


Since $f \in \Lambda_{W}^{2}$, Proposition 3.9 implies $\mathcal{F}_{W}[f](\omega) \in L_{W}^{2}\left(\mathbb{R}^{d}\right)$. Corollary 3.5 and equation (3.5) imply that in $L_{W}^{2}\left(\mathbb{R}^{d}\right)$,

$$
\mathcal{F}_{W}[f](\omega) e^{i \omega \cdot u}=\sum_{k=0}^{\infty}\left[\mathbb{K}^{k}[f](u)\right]^{T} \mathbb{P}_{k}(\omega) .
$$

Thus, equation (3.13) implies

$$
f(z)=\int_{\mathbb{R}^{d}} \sum_{k=0}^{\infty}\left[\mathbb{K}^{k}[f](u)\right]^{T} \mathbb{P}_{k}(\omega) e^{i\langle\omega, z-u\rangle} W(\omega) d \omega .
$$

Consequently, from (3.15) and (3.16),

$$
\begin{aligned}
\mid f(z) & -\sum_{k=0}^{n}\left[\mathbb{K}^{k}[f](u)\right]^{T} \Psi_{k}(z-u) \mid \\
& \leq \int_{\mathbb{R}^{d}}\left|\sum_{k=n+1}^{\infty}\left[\mathbb{K}^{k}[f](u)\right]^{T} \mathbb{P}_{k}(\omega) e^{i\langle\omega, z-u\rangle}\right| W(\omega) d \omega \\
& \leq\left(\int_{\mathbb{R}^{d}}\left|\sum_{k=n+1}^{\infty}\left[\mathbb{K}^{n}[f](u)\right]^{T} \mathbb{P}_{n}(\omega)\right|^{2} W(\omega) d \omega \int_{\mathbb{R}^{d}}\left|e^{i\langle\omega, z-u\rangle}\right|^{2} W(\omega) d \omega\right)^{1 / 2} .
\end{aligned}
$$

Since $u$ is real we have $\left|e^{i\langle\omega, z-u\rangle}\right|=\left|e^{\omega . \Im z}\right| \leq e^{\|\omega\|\|\Im z z\|}$. Thus, for $z \in S\left(\frac{c}{2}-\varepsilon\right)$ we have

$$
\begin{aligned}
\mid f(z) & -\sum_{k=0}^{n}\left[\mathbb{K}^{k}[f](u)\right]^{T} \Psi_{k}(z-u) \mid \\
& \leq\left(\sum_{k=n+1}^{\infty} \sum_{|\alpha|=n}\left|K_{\alpha}^{n}[f](u)\right|^{2} \int_{\mathbb{R}^{d}} e^{(c-2 \varepsilon)\|\omega\|} W(\omega) d \omega\right)^{1 / 2} .
\end{aligned}
$$

The claim now follows from Corollary 3.5 and (2.2).

\section{EXAmples}

The most efficient system of orthogonal polynomials to use in order to generate chromatic derivatives will depend on the shape of the support of the Fourier transform $\mathcal{F}_{W}[f]$ of $f$. Below we give some examples:

1 ) If the support of $\mathcal{F}_{W}[f]$ is the entire space $\mathbb{R}^{d}$, we use the $d$-dimensional Hermite polynomials. If we denote the normalized Hermite polynomial of degree $n$ by $H_{n}(x)$ so that

$$
\int_{\mathbb{R}} H_{m}(x) H_{n}(x) e^{-x^{2}} d x=\delta_{m, n},
$$

then the $d$-dimensional Hermite polynomial of degree $n$ is defined as

$$
H_{\alpha}(x)=H_{\alpha_{1}}\left(x_{1}\right) \cdots H_{\alpha_{d}}\left(x_{d}\right), \quad|\alpha|=n,
$$

with weight function $W(x)=e^{-\|x\|^{2}}, x \in \mathbb{R}^{d}$.

2) If the support of $\mathcal{F}_{W}[f]$ is the half space $\left\{x \in \mathbb{R}^{d}: x_{1}, \geq 0, \ldots, x_{d} \geq 0\right\}$, we use the normalized Laguerre polynomials

$$
L_{\alpha}(x)=L_{\alpha_{1}}^{k_{1}}(x) \cdots L_{\alpha_{d}}^{k_{d}}(x), \quad|\alpha|=n, x \in \mathbb{R}_{+}^{d}, k_{i} \geq-1,
$$


with weight function $W(x)=x^{k} e^{-|x|_{1}}$, where $|x|_{1}=x_{1}+\cdots+x_{d}, k=\left(k_{1}, \ldots, k_{d}\right)$ and $L_{n}^{\alpha}(x)$ is the normalized Laguerre polynomial of degree $n$ and parameter $\alpha$.

3 ) If the support of $\mathcal{F}_{W}[f]$ is the hypercube $D=[-1,1]^{d}$, we may use the $d$ dimensional Jacobi polynomials

$$
P_{\alpha}^{(a, b)}(x)=P_{\alpha_{1}}^{\left(a_{1}, b_{1}\right)}\left(x_{1}\right) \cdots P_{\alpha_{d}}^{\left(a_{d}, b_{d}\right)}\left(x_{d}\right), \quad|\alpha|=n,
$$

with weight function

$$
W^{(a, b)}(x)=\prod_{i=1}^{d}\left(1-x_{i}\right)^{a_{i}}\left(1+x_{i}\right)^{b_{i}},
$$

where $a=\left(a_{1}, a_{2}, \ldots, a_{d}\right), b=\left(b_{1}, b_{2}, \ldots, b_{d}\right)$ and $P_{n}^{(\alpha, \beta)}(x)$ is the normalized Jacobi polynomial of degree $n$ with parameters $\alpha, \beta$.

\section{Specialized two dimensional examples}

It should be noted that the theory of chromatic derivatives in higher dimensions raises more interesting possibilities than in one dimension because of the geometry that depends on the shape of support of $\mathcal{F}_{W}[f]$. We shall illustrate this by examples in two dimensions. But first, let us introduce the following method, which is due to T. Koornwinder [3], in order to generate orthogonal polynomials of two variables; see also 4 for another system of orthogonal polynomials in two variables.

Let $w_{1}$ and $w_{2}$ be two weight functions on the interval $(a, b)$ and $(c, d)$ respectively. Let $\rho$ be a positive function on $(a, b)$ such that either (i) $\rho$ is a polynomial of degree one or (ii) $\rho$ is the square root of a non-negative polynomial of degree at most two, and assume that the interval $(c, d)$ is symmetric around the origin, i.e., it is in the form $(-c, c), c>0$ and $w_{2}$ is even on $(-c, c)$.

For each $k \in N_{0}$, let $\left\{p_{n, k}\right\}_{n=0}^{\infty}$ denote the system of orthonormal polynomials with respect to the weight function $\rho^{2 k+1}(x) w_{1}(x)$. Let $\left\{q_{n}\right\}_{n=0}^{\infty}$ be a system of orthonormal polynomials with respect to $w_{2}$. Define

$$
P_{n}^{k}(x, y)=p_{n-k, k} \rho^{k}(x) q_{k}\left(\frac{y}{\rho(x)}\right), \quad 0 \leq k \leq n .
$$

Then $P_{k}^{n}(x, y)$ are orthonormal polynomials with respect to the weight function

$$
W(x, y)=w_{1}(x) w_{2}(y / \rho(x)), \quad(x, y) \in \mathbb{R},
$$

on the domain

$$
D=\{(x, y): a<x<b, c \rho(x)<y<d \rho(x)\} .
$$

Therefore, by changing the variables, we have

$$
\begin{aligned}
& \iint_{D} P_{n}^{k}(x, y) P_{m}^{j}(x, y) W(x, y) d x d y \\
& =\int_{a}^{b} p_{n-k, k} p_{m-j, j} \rho^{k+j+1}(x) w_{1}(x) d x \int_{c}^{d} q_{k}(y) q_{j}(y) w_{2}(y) d y \\
& =\delta_{m, n} \delta_{k, j} .
\end{aligned}
$$

Examples. 1) If the support of $\mathcal{F}_{W}[f]$ is the unit disc, we take $w_{1}(x)=w_{2}(x)=$ $\left(1-x^{2}\right)^{\lambda-1 / 2}$ on $[-1,1]$, and $\rho(x)=\left(1-x^{2}\right)^{1 / 2}$. The weight function according to (5.1) is

$$
W(x, y)=\left(1-x^{2}-y^{2}\right)^{\lambda-1 / 2}
$$


on the domain $D=\left\{(x, y): x^{2}+y^{2} \leq 1\right\}$. We have

$$
P_{n}^{k}(x, y)=C_{n-k}^{\lambda+k+1 / 2}(x)\left(1-x^{2}\right)^{k / 2} C_{k}^{\lambda}\left(y / \sqrt{1-x^{2}}\right),
$$

where $C_{n}^{k}(x)$ is the Gegenbauer polynomial of degree $n$ and parameter $k \geq-1 / 2$.

2) If the support of $\mathcal{F}_{W}[f]$ is the triangle $D=\{(x, y): x, y \geq 0,1-x-y \geq 0\}$, we choose $w_{1}(x)=x^{\alpha-1 / 2}(1-x)^{\beta+\gamma-1}$ and $w_{2}(x)=x^{\beta-1 / 2}(1-x)^{\gamma-1}$ defined on $(0,1)$ and $\rho(x)=1-x$. The weight function according to (5.1) is

$$
W(x, y)=x^{\alpha-1 / 2} y^{\beta-1 / 2}(1-x-y)^{\gamma-1 / 2} .
$$

The orthonormal polynomials $P_{n}^{k}(x, y)$ are easily seen to be

$$
P_{n}^{k}(x, y)=P_{n-k}^{\left(a_{1}, b_{1}\right)}(2 x-1)(1-x)^{k} P_{k}^{\left(a_{2}, b_{2}\right)}\left(2 \frac{y}{\rho}-1\right),
$$

where

$$
a_{1}=\alpha-1 / 2, b_{1}=\beta+\gamma+2 k, a_{2}=\beta-1 / 2, b_{2}=\gamma-1 / 2 .
$$

3) If the support of $\mathcal{F}_{W}[f]$ is the parabola $D=\{(x, y):-\sqrt{x} \leq y \leq \sqrt{x} \leq 1\}$, we take $w_{1}(x)=x^{\alpha}(1-x)^{\beta}$ on $[0,1]$ and $w_{2}(x)=\left(1-x^{2}\right)^{\alpha}$, and $\rho(x)=\sqrt{x}$. The weight function according to (5.1) is

$$
W(x, y)=(1-x)^{\beta}\left(x-y^{2}\right)^{\alpha},
$$

and the orthonormal polynomials are

$$
P_{n}^{k}(x, y)=P_{n-k}^{(\alpha+k+1 / 2, \beta)}(2 x-1) x^{k / 2} C_{k}^{\alpha+1 / 2}\left(\frac{y}{\sqrt{x}}\right),
$$

where $P_{n}^{(a, b)}(x)$ is the normalized Jacobi polynomial of degree $n$ and $C_{n}^{\lambda}(x)$ is the Gegenbauer polynomial of degree $n$.

\section{REFERENCES}

1. C. Dunkl and Y. Xu, Orthogonal Polynomials of Several Variables, Encyclopedia of Mathematics, Cambridge University Press, 2001. MR1827871 (2002m:33001)

2. H. Krall and I. Sheffer, Orthogonal polynomials in two variables. Ann. Mat. Pura Appl. (4) 76 (1967), 325-376. MR0228920 (37:4499)

3. T. Koornwinder, Two-variable analogues of the classical orthogonal polynomials, in Theory and Applications of Special Functions, pp. 435-495, ed. R. Askey, Academic Press, New York, 1975. MR0402146 (53:5967)

4. T. Koornwinder, Orthogonal polynomials in two variables which are eigenfunctions of two algebraically independent partial differential operators, I, II, Proc. Kon. Akad. v. Wet., Amsterdam (36) (1974), 48-58, 59-66. MR:0340673 (49:5425a)

5. A. Ignjatovic, Numerical Differentiation and Signal Processing, Kromos Technology technical report, Los Altos, California, 2001.

6. A. Ignjatovic, Numerical differentiation and signal processing, Proc. International Conference on Information, Communications and Signal Processing (ICICS), Singapore, 2001.

7. A. Ignjatovic, Local approximations based on orthogonal differential operators, Journal of Fourier Analysis and Applications, Vol. 13, No. 3, 2007. MR2334612 (2008i:41038)

8. A. Ignjatovic, Signal Processor with Local Signal Behavior, U.S. Patent 6115726 filled October 1997, issued September 2000.

9. A. Ignjatovic and N. Carlin, Method and a system of acquiring local signal behavior parameters for representing and processing a signal, U.S. Patent 6313778, filled July 1999, issued November 6, 2001.

10. A. Ignjatovic, Chromatic derivatives and local approximations, IEEE Transactions on Signal Processing, Vol. 57, No. 8, 2009. MR 2723042

11. A. Ignjatovic, Chromatic derivatives, chromatic expansions and associated spaces, East Journal on Approximations, Vol. 15, No. 3, 2009, pp. 263-302. MR.2741822 
12. M. Cushman and T. Herron, The General Theory of Chromatic Derivatives, Kromos Technology technical report, Los Altos, California, 2001.

13. M. J. Narasimha, A. Ignjatovic and P. P. Vaidyanathan, Chromatic derivative filter banks, IEEE Signal Processing Letters, Vol. 9, No. 7, 2002.

14. P. P. Vaidyanathan, A. Ignjatovic and S. Narasimha, New Sampling Expansions of Band Limited Signals Based on Chromatic Derivatives, Proc. 35th Asilomar Conference on Signals, Systems, and Computers, Monterey, California, 2001.

15. G. Walter and X. Shen, A Sampling Expansion for Nonbandlimited Signals in Chromatic Derivatives, IEEE Transactions on Signal Processing, Vol. 53, 2005. MR2128248(2005j:94012)

16. G. Walter, Chromatic series and prolate spheroidal wave functions, Journal of Integral Equations and Applications, Vol. 20, No. 2, 2006. MR2418070 (2010b:41025)

17. A. Zayed, Advances in Shannon's Sampling Theory, CRC Press, Boca Raton, Florida, 1993. MR 1270907 (95f:94008)

18. A. Zayed, Generalizations of Chromatic Derivatives and Series Expansions, IEEE Transactions of Signal Processing, Vol. 58, Issue 3, 2010, pp. 1638-1647. MR2730105

School of Computer Science and Engineering, University of New South Wales, SydNeY, Australia

E-mail address: ignjat@cse.unsw.edu.au

Department of Mathematical Sciences, DePaul University, Chicago, Illinois 60614

E-mail address: azayed@condor.depaul.edu 